ARGONNE NATIONAL LABORATORY

Argonne, Illinois

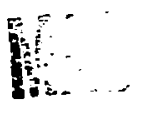

\title{
A ZGS BEAM TRANSPORT FOR TRANSVERSE OR \\ LONGITUDINALLY POLARIZED PRO'TONS*
}

Eugere Colton, I. Paul Auer, Andrew Beretvas, Dan Hill, Kenneth Nield, Bernard Sandler, Harold Spinka, David Underwood, Yasushi Watanabe, and Akihiko Yokosatwa
- NOTICE

Ths repan was prepared as an accauns of werk sponsored to the tinsted Surtes Governisem Nesthet

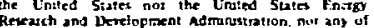

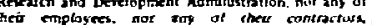

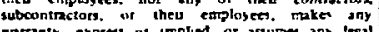

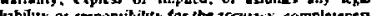

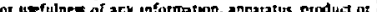

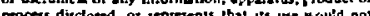
infringe pnsateli; wened rights

August 11, 1977

Accelerator Research Facilities Division Accelerator Report

ACC - 3

*Work performed under the auspices of the United States Energy Research and Development Administration. 
The facilities of Argonne National Laboratory are owned by the United States Government. Under the terms of a contract (W-31-109-Eng-38) between the U. S. Energy Research and Development Administration, Argonne Universities Association and The University of Chicago, the University employs the staff and operates the Laboratory in accordance with policies and programs formulated, approved and reviewed by the Association.

\section{MEMBERS OF ARGONNE UNIVERSITIES ASSOCIATION}

The University of Arizona

Carnegie-Mellon University

Case Western Reserve University

The University of Chicago

University of Cincirnati

Illinois Institute of Technology

Unive rsity of Illinois

Indiana University

Iowa State University

The University of Iowa
Kansas State University The University of Kansas Loyola University Marquette University Michigan State University The University of Michigan University of Minnesota University of Missouri Northwestern University University of Notre Dame
The Ohio State University

Ohio University

The Pennsylvania State University

Purdue University

Saint Louis University

Southern Illinois University

The University of Texas at Austin

Washington University

Wayne State University

The University of Wiscorsin

\section{NOTICE}

This report was prepared as an account of work sponsored by the United States Government. Neither the United States nor the United States Energy Research and Development Administration, nor any of their employees, nor any of their contractors, subcontractors, or their employees, makes any warranty, express or implied, or assumes any legal liability or responsibility for the accuracy, completeness or usefulness of any information, apparatus, product or process disclosed, or represents that its use would not infringe privataly-owned rights. Mention of commercial products, their manufacturers, or their suppliers in this publication does not imply or connote approval or disapproval of the product by Argonne National Laboratory or the U. S. Energy Research and Development Administration. 


\section{ARGONNE NATIONAL LABORATORY}

Argonne, Illinois

A ZGS BEAM TRANSPORT FOR TRANSVERSE OR

LONGITUDINALLY POLARIZED PROTONS*

Eugene Colton, I. Paul Auer, Andrew Beretvas, ${ }^{\dagger}$ Dan Hi11, Kenneth Nield, Bernard Sandier, ${ }^{++}$Harold Spinka, David Underwood, Yasushi Watanabe, and Akihiko Yokosawa

August 11, 1977

Accelerator Research Facilities Division

Accelerator Report

$\mathrm{ACC}-3$

ABSTRACT

A combination of dipole magnets and superconducting solenoid is utilized to transform the spin direction of transversely polarized protons from the Argonne $\mathrm{ZGS}$ for use in proton-proton scattering experiments.

† Present address: Fermi National Accelerator Laboratory, Batavia, Illinois 60510, U.S.A.

t+ Present address: Randal1 Laboratory of Physics, University of Michigan, Ann Arbor, Michigan 48104, U.S.A.

* Work performed under the auspices of the United States Energy Research and Development Administration. 
The recent successful acceleration and extraction of polarized protons from the Argonne Zero Gradient Synchrotron ${ }^{3}$ (ZGS) has opened up a whole new realm of high energy physics research into the proton-proton interaction. Experiments previously considered impract ical are now being performed: Doubleand triple-spin measurements are routinely carried out using the polarized beam, polarized proton targets (PPT's), and recoil polarimeters. ${ }^{2}$ One facility (PPT-III) uses a superconducting 2.5T "R and A" magnet. The magnet (and target) can be rotated about a vertical axis and it produces polarization in the horizontal plane; thus, the target polarization can be made to point along the incoming beam direction (longitudinal or $L$ direction) or transverse to it (S direction). ${ }^{3}$ Recently, several interesting measurements were performed using the target in the L-type configuration. ${ }^{4}$ In this case, we utilized a modified existing transport line (Beam 22B) to provide longitudinally polarized incident protons. The beam line has been subsequently taken through several iterations; now it can deliver to PPT-III proton beams of momenta up to $6 \mathrm{GeV} / \mathrm{c}$ and with polarization pcinting in any desired direction. However, we normally tune the beam line to compensate for the additional precession due to the field of the "R and A" magnet, and obtain a beam polarization at the center of the target which points in the $S, L$, or $N$ (vertical) direction. We discuss this beam transport in the remainder of this paper.

The protons incident to Beam 22B are first accelerated in the ZGS, extracted, and then transported down an existing external proton beam line (EPB-I); an $\mathrm{N}$-type beam polarization is maintained through these steps. The protons are then focussed onto a $0.6 \mathrm{~m}$ long hole collimator which acts as the source for Beam 22B. Typically, we accept an incident beam size of 0.6 $1.3 \mathrm{~cm}$ diameter, beam divergences of $\pm 3 \mathrm{mrad}$, and intensities of $10^{5}-10^{6}$ protons per pulse. As shown in Fig. 1(a), the first stage of Beam 22B is composed of a magnetic quadrupole doublet (Q1-Q2) air sever:1 dipole magnets; 
these serve to transport, and image the source at the first focus (f1). At this point, the magnifications are typically -2.5 and -0.5 , in the horizontal a. 1 vertical planes, respectively. ${ }^{5}$ The optical layout of the second stage of the beam is displayed in Fig. 1(b). The two doublets (Q3-Q4 and Q5-Q6) serve to recapture, transport, and refocus the beam at the PFT-III location (f2 image); the magnifications are 0.7 and 0.5 , in the horizontal and vertical planes, respectively. The bending (dipole) magnets (B2, B3, and B4) are positioned and powered so as to preserve fixed beam directions into B4, and into f2. Furthermore, if an N-type beam is desired, then the solenoid is not powered. The solenoid is utilized if either an S- or L-type beam is desired at the PPT. The superconducting solenoid depicted in Fig. 1(b) has a maxinum field strength $\int \vec{B}_{S} \cdot \vec{d}_{\ell}=120 \mathrm{kG}-\mathrm{m}$. This magnet is a monolithic winding ith a cold bore of $11.2 \mathrm{~cm}$, and effective length of $2.0 \mathrm{~m}$. The conductor is $\mathrm{NbTi}$ stablized with copper, and the maximm central field is $67 \mathrm{kG}$. The heat gain to the helium vessel is $1.3 \mathrm{~W}$ at 400 amperes. The solenoid has never quenched over several month-long running periods. When the solenoid is powered, the spin axis of the incident protons ( $N$ type) is precessed about the beam direction because the proton magnetic moment $(\mathrm{g} / 2 \equiv 2.7928$ nuclear magnetons) interacts with the axial magnetic field. The relation between the field strength and spin rotation angle of $\phi$ degrees is given by

$$
\int \vec{B}_{S} \cdot d \vec{l}=\frac{0.582 P \phi}{g / 2}
$$

where $P$ is the beam momentum in GeV/c. ${ }^{7}$ The spin rotation angle must be \pm $90^{\circ}$ in order to transform the incident proton spin direction from $\mathrm{N}$ type to $S$ type. If we require an S-type beam at the PPT, then B4 is turned off. Otherwise, we utilize $B 4$ to precess the proton spins by $90^{\circ}$ into the $L$ direction; in this case the bend angle of $\mathrm{B} 41 \mathrm{~s}$ given by ${ }^{7}$ 


$$
\theta_{4} \text { (degrees) }=\frac{90}{\left(\frac{g}{2}-1\right) \gamma}
$$

where $\gamma=\sqrt{\mathrm{P}^{2}+\mathrm{m}^{2}} / \mathrm{m}$ with $\mathrm{m}=0.9382 \mathrm{GeV}$. The setting for $\mathrm{B} 4$ was determined by the wire-orbit method.

Equation (2) indicates that the bend angle of $\mathrm{B} 4$ is different for each momentum when ore utilizes an L-type beam. The bend angles of B2 and B3 must also be changed in order to satisfy the constraints quoted above. Operationally, this means that the physical bend point of $\mathrm{B} 3$, and the solenoid are moved for each change in momentum; aperture limitations sometimes require repositioning B2 and/or B3. The geometrical layout of the bend points and angles is disnlayed in Fig. 2; the origin is at the B2 bend point with the undeflected line chosen along the $+\mathrm{Z}$ axis. We show two positions for the B3 bend point in Fig. 2: (a) B3(S) is the S- or N-type beam position $\left(z_{3 S}, x_{3 S}\right)$ with bend angles $\theta_{2 S}$ and $\theta_{3 S} ;\left(\right.$ (b) $B 3(L)$ is an L-type beam position $\left(Z_{3 L}, X_{3 L}\right)$ with bend angles $\theta_{2 \mathrm{~L}}$ and $\theta_{3 \mathrm{~L}}$. In order to determine the parameters as shown in Fig. 2, we must specify $\theta_{T}$ (the angle into the PPT), the PPT location $\left(\mathrm{z}_{\mathrm{T}}, \mathrm{X}_{\mathrm{T}}\right)$, and the distances between the $\mathrm{B} 3$ and $\mathrm{B} 4$ bend points $[\mathrm{D}(34)]$, and $\mathrm{B} 4$ bend point and PPT $[\mathrm{D}(4 \mathrm{~T})]$. The magnet bend points are given by

$$
\begin{aligned}
& z_{4}=z_{T}-D(4 T) \cos \theta_{T} \\
& z_{3 S}=z_{4}-D(34) \cos \theta_{T} \\
& z_{3 L}=z_{4}-D(34) \cos \left(\theta_{T}-\theta_{4}\right) \\
& x_{4}=x_{T}-D(4 T) \sin \theta_{T} \\
& x_{3 S}=x_{4}-D(34) \sin \theta_{T} \\
& x_{3 L}=x_{4}-D(34) \sin \left(\theta_{T}-\theta_{4}\right)
\end{aligned}
$$


Similarly, we calculate the bend angles of B2 and B3:

$$
\begin{aligned}
& \theta_{2 S}=\arctan \left(\frac{X_{3 S}}{z_{3 S}}\right) \\
& { }_{3 S}=\theta_{T}-\theta_{2 S} \\
& { }_{2 L}=\arctan \left(\frac{X_{3 L}}{z_{3 L}}\right) \\
& { }_{3 L}=\theta_{2 L}+\theta_{4}-\theta_{T}
\end{aligned}
$$

During the running of an experiment, multiwire proportional chambers with $2 \mathrm{~mm}$ wire spacing are utilized in order to monitor the beam conditions. In particular, the maintenance of a constant $\theta_{4}$ is crucial to ensure that improper spin precession does not occur (for an L-type beam). Accordingly, we monitor the horizontal beam profiles at the upstream and downstream ends of the solenoid; a typical oscilloscope trace of the beam profiles at the ends of the solenoid is shown in Fig. 3(a). We use magnets B2 and B3 to keep the beam centered on-axis going through the solenoid (and centered on the wire chambers). Beam conditions are also closely monitored at the entrance to the PPT: Figures 3 (b) and 3 (c) show, respectively, the horizontal and vertical beam profiles. The beam sizes at the PPT are quite small $(0.8-1.0 \mathrm{~cm}$ FWHM $)$ in both transverse planes.

During an initial phase of operating the beam line and target in the L-type configuration at $\dot{\mathbf{p}}=1.47 \mathrm{GeV} / \mathrm{c}$, we swept the solenoid current so as to cover spin rotation angles $0^{\circ} \leq \phi \leq 180^{\circ}$. In Fig. 4 we display the measured difference in the total cross sections $\Delta \sigma_{L}$ for the beam spin in opposite directions, plotted as a function of $I / I_{0}$ where $I$ is the solenoid current and $I_{0}$ is the calculated current required to produce a spin rotation 
of $90^{\circ}\left(1 / I_{0} \equiv \phi / 90^{\circ}\right)$. The quantity $\Delta \sigma_{L}$ should be proportional to the $L$ component of beam spin, i.e., $\Delta \sigma_{L} \alpha \sin \phi$. The curve $-10.5 \sin \left[(\pi / 2) \cdot\left(I / I_{0}\right)\right]$ is plotted for comparison in Fig. 4: it verifies the expected shape of $\Delta \sigma_{L}$ and the solenoid calibration.

Depolarization of the beam through the solenoid due to non-axial momentum components is estimated to be less than $1 \%$. Depolarization due to quadrupoles is assumed to cancel to first order since the beam spatial magnifications are positive at the PPT.

Many people have contributed to the successful construction, testing, and operation of this general purpose beam line. In particular, we thank Dr. R. Klem for designing the original Beam 22B line, and C. Brzegowy, D. Gacek, and F. Onesto for engineering support. The personnel of the Accelerator Research Facilities Division are to be commended for carrying out a successful installation and maintenance of the modified beam line. 


\section{REFERENCES}

1. For a discussion of polarized proton acceleration in the ZGS, see e.g., T. Khoe,et a1., Particle Accelerators 6, 213 (1975).

2. See e.g., A. D. Krisch, High Energy Experiments with a Polarized Bean and Target, Lecture presented at 10th LAMPF Users Meeting, Los Alamos, N. M. (November 8, 1976).

3. The orthogonal coordinate system is defined by $\hat{S}=\hat{N} \times \hat{L}$ where $\hat{L}$ represents the incoming beam direction and $\hat{N}$ is transverse to $\hat{L} ; \hat{N}$ points either up or down.

4. I. P. Auer, et a1., Physics Letters, 67B, 113 (1977). See also e.g., I. P. Auer, A. Beretvas, E. Colton, D. Hil , K. Nield, H. Spinka D. Underwood, Y. Watanabe, and A. Yokosawa, Argonne Report ANL-HEPPR-77-29.

5. Quadrupole Strengths are determined Using a Beam Program Such as TRANSPORT, see e.g., K. L. Brown, D. C. Carey, Ch. Iselin, and F. Rothacker, CERN 73-16 (1973).

6. Manufactured by American Magnetics, Inc., Oak Ridge, Tenn.

7. See e.g., A. P. Banford, The Transport of Charged Particle Beams, Sect. 7.4, E. and F. N. Spon Limited (London, 1966). 


\section{FIGURE CAPTIONS}

Fig. 1(a) Horizontal plane optical layout of the first stage of Beam 22 SB represents several short magnets which bend the beam throu $5.5^{\circ}$. Similarly, B1 is a rectangular bending magnet which produces a $7^{\circ}$ bend.

(b) Horizortal plane optical layout of the second stage of Beam 22B. Magnets B2, B3, and B4 are rectangular bending magnets. The solenoid is off for transport of N-type beams; B4 is off for transport of an S-type beam.

Fig. 2

Geometrical layout of bend points of B2, B3, and B4 along with the PPT location. The undeflected line (B2 off) travels in the +2 direction. See text for definition of angles and distances.

Fig. 3(a) Horizontal plane profiles at the solenoid ends obtained using integrating multiwire proportional chambers with $2 \mathrm{~nm}$ wire spacing. The top trace was taken at the upstream end, and the bottom trace at the downstream end. A dead wire is evider in the lower distribution.

(b) (c) Horizontal and vertical profiles at the entrance to the PPT obtained using a multiwire proportional chamber with 2 mn wire spacing.

Proton-proton total cross-section difference between incident spin states aligned antiparalle1, and parallel to the beam direction $\Delta \sigma_{L}$, measured as a function of the solenoid spin 
(a)

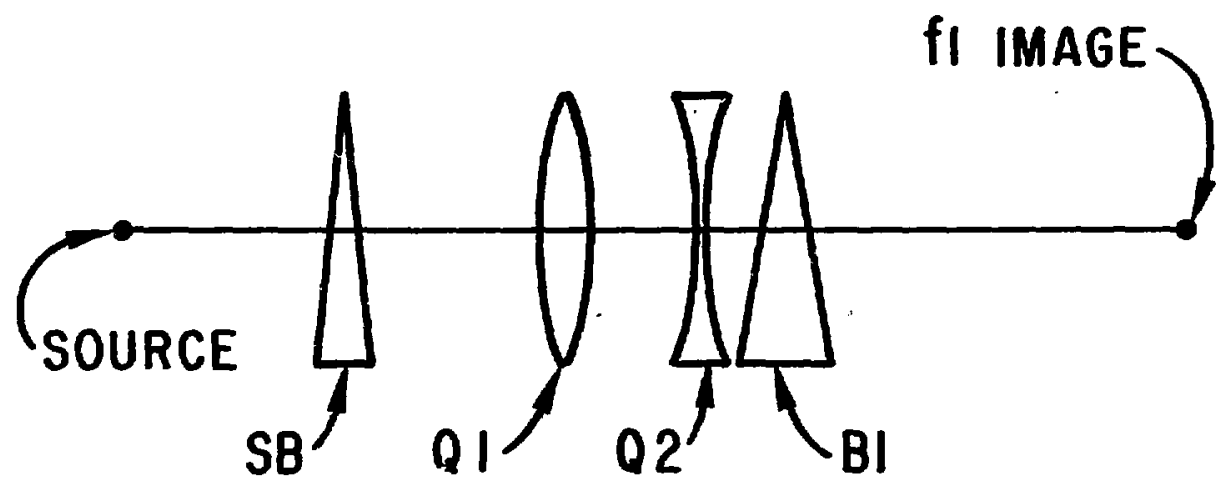

(b)

SOLENOID, $\quad 5$

fI IMAGE

f2 IMAGE

(PPT)

Figure 1 


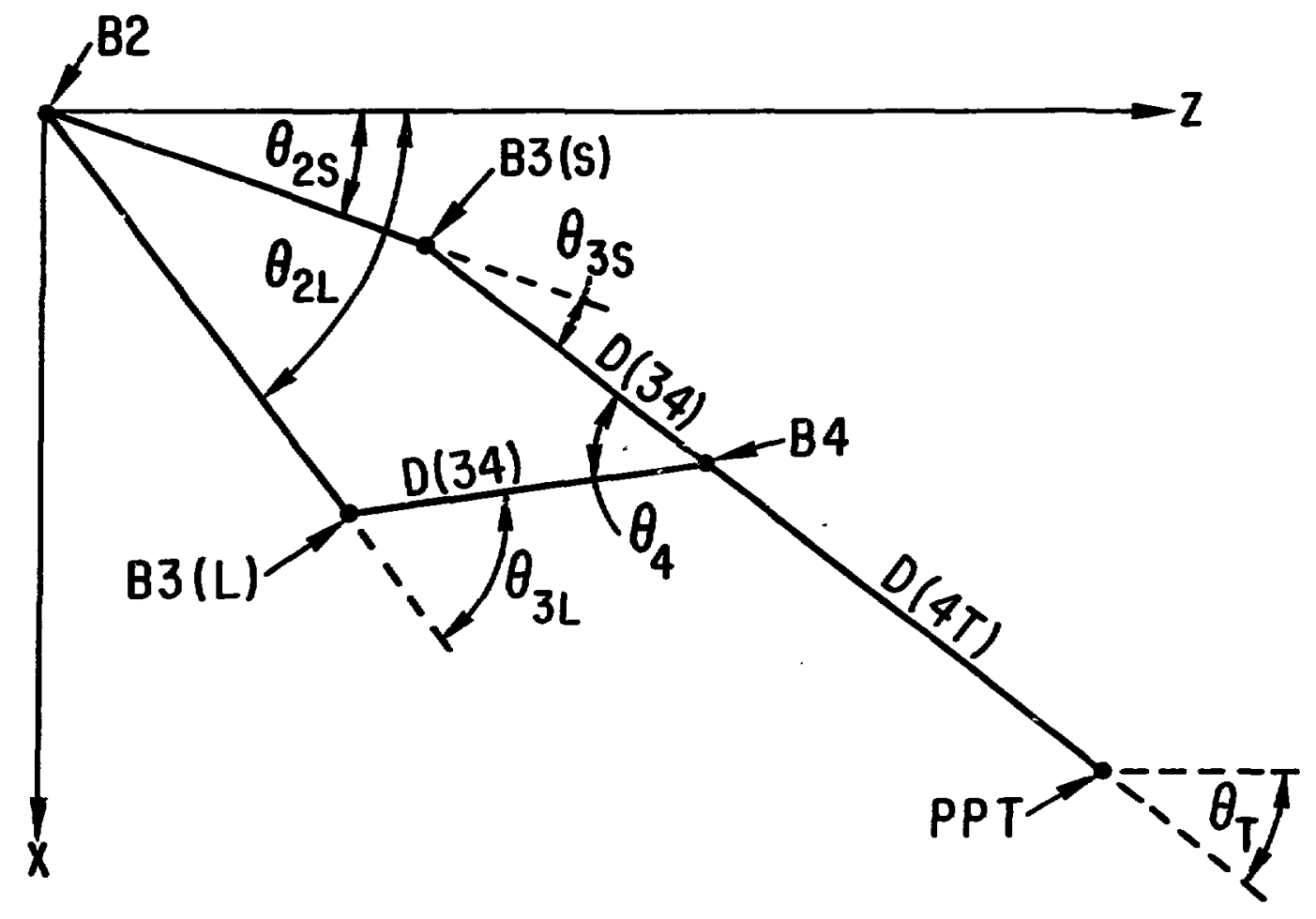

Figure 2 
(a)
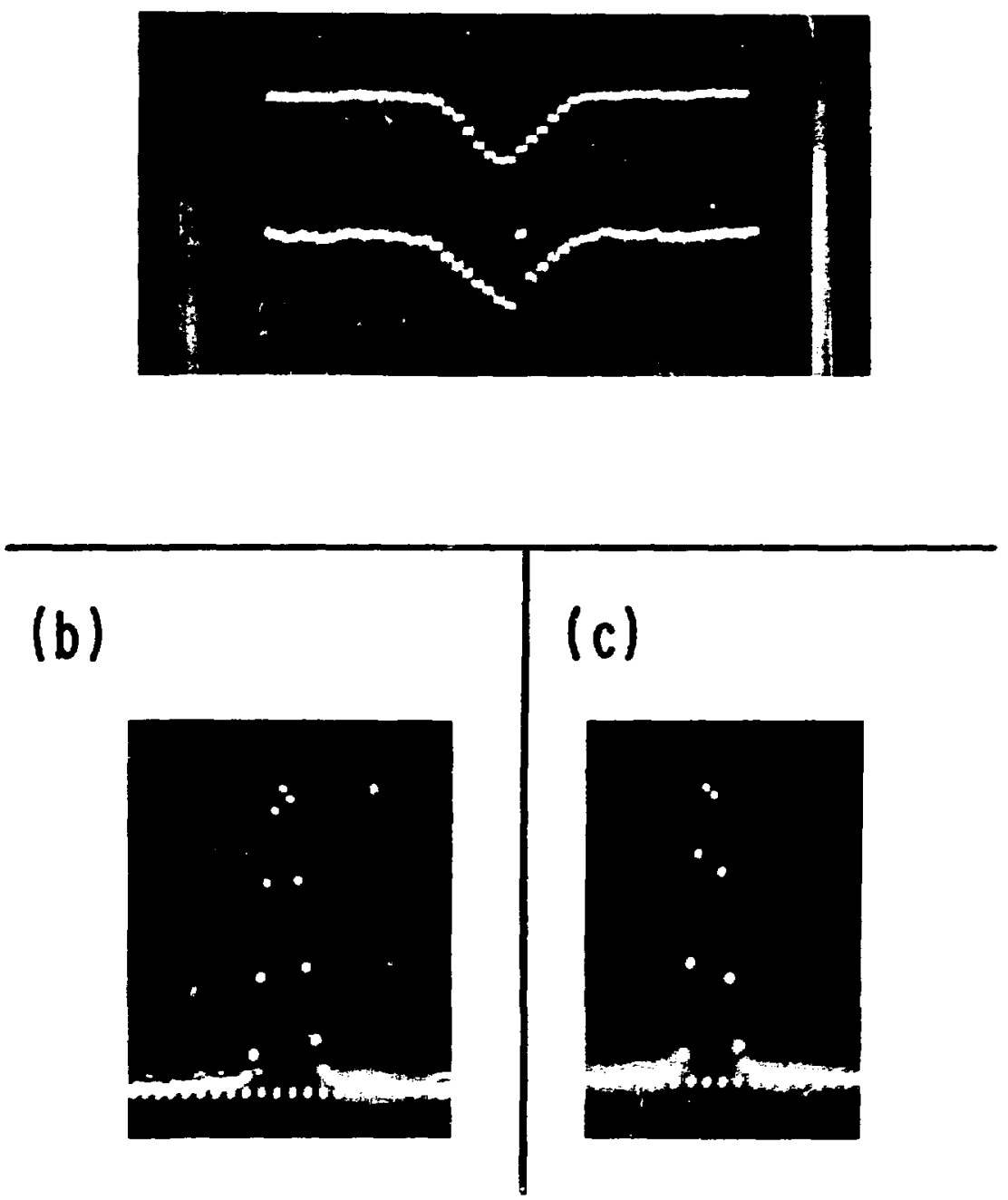

Figure 3 


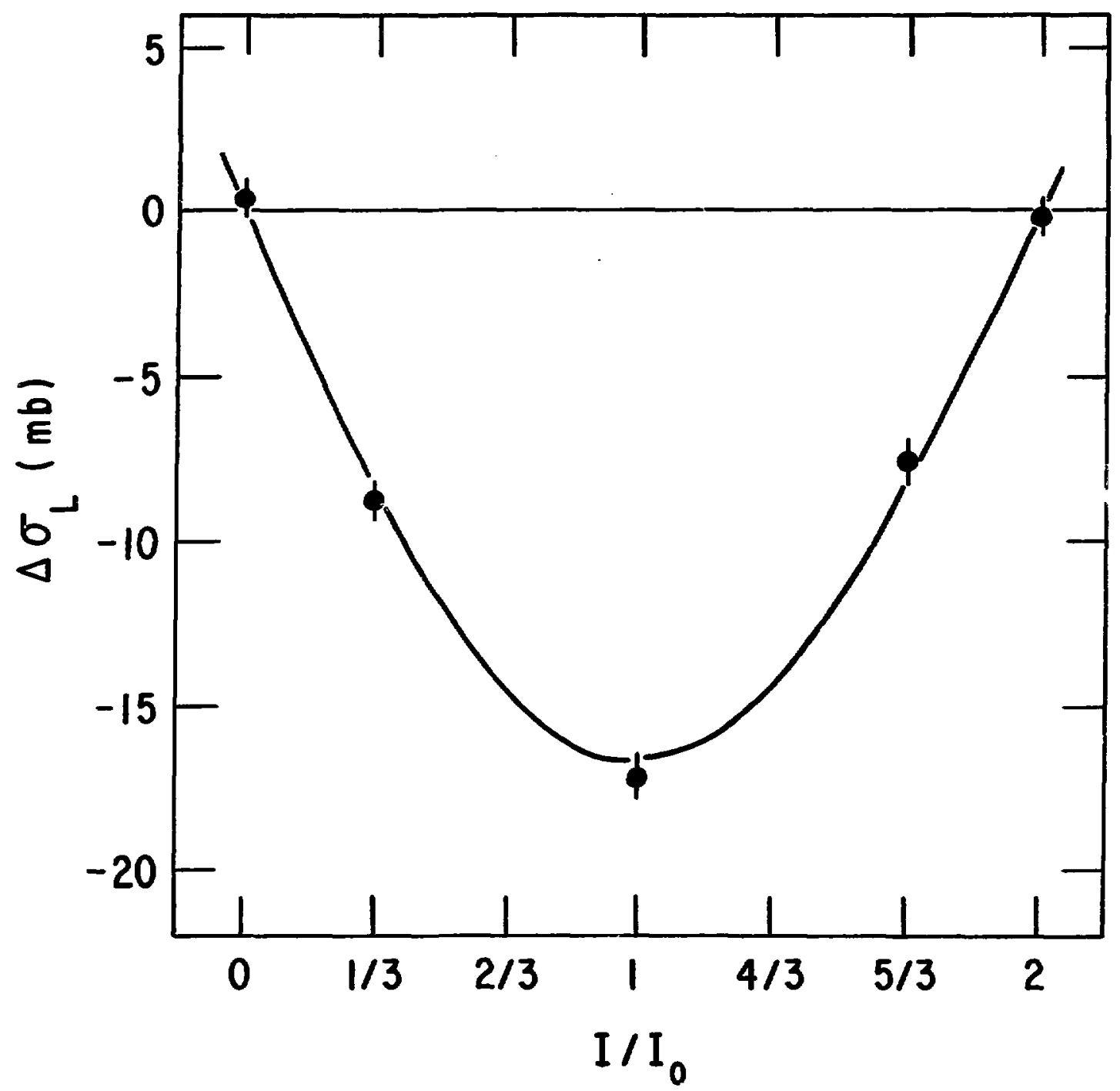

Figure 4 